\title{
Análisis de polimorfismos en los genes tripanotión reductasa y cruzipaína en cepas colombianas de Trypanosoma cruzi
}

\author{
Winston Rojas ${ }^{1}$, Maria Antonieta Caro ${ }^{1}$, Juan Guillermo Lopera ${ }^{1}$, Omar Triana , \\ Juan Carlos Dib 2, Gabriel Bedoya ${ }^{1}$ \\ ${ }^{1}$ Genética Molecular, Universidad de Antioquia, Medellín, Colombia. \\ ${ }^{2}$ Grupo de Chagas, Universidad de Antioquia, Medellín, Colombia.
}

Introducción. Los estudios genéticos en Trypanosoma cruzi han buscado establecer asociaciones de variantes genéticas del parásito con manifestaciones clínicas de la enfermedad, origen biológico y geográfico de los aislamientos; sin embargo, los resultados de asociación con los marcadores comúnmente usados en estos estudios han generado mucha controversia, principalmente en la asociación de grupos con características clínicas y epidemiológicas de la enfermedad.

Objetivo. Se planteó determinar la variabilidad de genes que codifican para las proteínas tripanotión reductasa y cruzipaína, involucradas en mecanismos de infección y supervivencia del parásito en el hospedador mamífero, y probar la asociación de variantes génicas con origen biológico y geográfico de cepas colombianas de $T$. cruzi.

Materiales y métodos. Se tipificaron por reacción en cadena de la polimerasa- polimorfismo de longitud del fragmento de restricción seis SNPs (Single Nucleotide Polymorphisms) en tripanotión reductasa y ocho SNPs en cruzipaína en 36 cepas colombianas de $T$. cruzi de diferentes regiones y origen biológico.

Resultados. Con las enzimas Acy I y Hae III se determinaron tres genotipos para tripanotión reductasa. Para cruzipaína se identificaron seis genotipos con las enzimas Rsa I, Ban I y Bsu 361 .

Conclusiones. Para tripanotión reductasa no fue posible establecer una asociación con el origen biológico o geográfico; sin embargo los alelos producidos en las posiciones 102 y 210, permitieron discriminar los grupos tradicionales I y II. Con los genotipos obtenidos para cruzipaína se establecieron relaciones a estos grupos, origen biológico y geográfico. Los resultados sugieren la utilidad de estos genes como marcadores moleculares para determinar y diferenciar variedades genéticas en $T$. cruzi.

Palabras clave: Trypanosoma cruzi, genes protozoarios, polimorfismo, polimorfismo de longitud del fragmento de restricción, Colombia.

Analysis of polymorphisms in the trypanothione reductase and cruzipain genes in Colombian strains of Trypanosoma cruzi

Introduction. Genetic studies of Trypanosoma cruzi have tried to establish relations between genetic variants and their biological characteristics, such as clinical manifestations, host or geographic origin. However, much controversy exists on the associations between the commonly used DNA markers with group, clinical characteristics and disease epidemiology.

Objective. In this study determined the variability of the genes that code for the proteins trypanothione reductase and cruzipain, both involved in the infection and survival of the parasite in the mammalian host, was studied and the association between genetic polymorphism and biological and geographic sources in Colombian T. cruzi strains was examined.

Materials and methods. The genotypes for each of six SNPs (single nucleotide polymorphisms) for trypanothione reductase and eight SNPs for cruzipain genes were identified by polymerase chain reaction-restriction fragment length polymorphism.in $36 \mathrm{~T}$. cruzi Colombian stocks from several regions and biological origins. 
Results. Three genotypes were identified for trypanothione reductase with Acy I and Hae III enzymes and six genotypes for cruzipain with the Rsa I, Ban I and Bsu 36I enzymes.

Conclusion. For trypanothione reductase , an association was not established with biological or geographical origin; however, alleles at positions 102 and 210 allowed discrimination with groups I and II. For cruzipain, specific genotypes were associated with group, biological and geographic origin. The usefulness of molecular markers on these genes was demonstrated for the determination and differentiation of genetic varieties in $T$. cruzi.

Key words: Trypanosoma cruzi, genes, protozoan polymorphism, restriction fragment length polymorphism; Colombia.

Trypanosoma cruzi, el flagelado que causa la enfermedad de Chagas, realiza su ciclo de vida entre reservorios mamíferos e insectos de la familia Reduviidae. Para este protozoario se ha propuesto un modo de reproducción predominantemente clonal (1), con lo cual se esperaría encontrar una baja diversidad. Sin embargo, al evaluar marcadores fenotípicos, morfológicos y bioquímicos (2), muchos estudios muestran, por el contrario, una alta heterogeneidad. Es razonable pensar que esta diversidad pueda estar influyendo, por lo menos en parte, en el rango variable de las manifestaciones clínicas de la enfermedad (2). Con este planteamiento se han emprendido estudios que buscan asociaciones entre la heterogeneidad genética del parásito y la heterogeneidad clínica de la enfermedad (3-9).

Con el uso de marcadores de ADN anónimos (Random Amplified Polymorphic DNA, RAPD) o en los genes kADN, espaciadores intergénicos del gen mini-exón, y en el ADN ribosomal se ha logrado determinar grupos y subgrupos de $T$. cruzi e incluso establecer un patrón regional en relación con estas divisiones (10-15). Sin embargo, hasta la fecha existe mucha controversia sobre las reales asociaciones entre los grupos y las características clínicas y epidemiológicas de la enfermedad, puesto que ambos grupos se han encontrado en todos los estados de transmisión, en diversas fases de la enfermedad y en sus diferentes rasgos fenotípicos (16). Es también razonable pensar que, de existir una asociación,

\footnotetext{
Correspondencia:

Winston Rojas, Calle 62 \# 52-59, Sede de Investigación Universitaria (SIU), 4 piso- 430, Medellín, Colombia. Tel. (4) 2106464, fax: (4) 2106469.

winstonrojas@yahoo.com
}

Recibido: 20/12/05; aceptado: 07/04/06 sería útil estudiar los genes involucrados en procesos como la infección de la célula hospedera, la supervivencia y la progresión del ciclo de vida del parásito, así como aquellos útiles en evadir la respuesta inmune del hospedero, podrían ser un blanco importante.

El genoma de $T$. cruzi consta de aproximadamente 22.570 genes, de los cuales casi el 50\% conforma grupos parálogos. Entre ellos se encuentra la cruzipaína (Crp), que pertenece a una familia de cisteín peptidasas compuesta por 20 miembros por genoma haploide (17). El porcentaje restante corresponde a genes de copia única como la tripanotión reductasa (TriR) (18).

Los tripanosomátidos carecen de la enzima glutatión peroxidasa y para defenderse del estrés oxidativo utilizan el tripanotión reducido en un sistema propio de estos organismos (19). Esta reducción es catalizada por la tripanotión reductasa, una flavoproteína dependiente de NADPH, la cual además participa en el metabolismo y detoxificación de drogas y en la reducción de precursores para la síntesis de ADN $(20,21)$. En Leishmania spp. este sistema está involucrado en la supervivencia intracelular del parásito (22). El gen que codifica para esta enzima también ha demostrado ser útil en la determinación de grupos dentro de T. cruzi, pues ha revelado la existencia de cuatro grupos principales en lugar de las dos divisiones ya conocidas (18).

La cruzipaína pertenece a la familia de las cisteín proteinasas, y es codificada por un alto número de genes organizados como grupos localizados en diferentes cromosomas $(23,24)$. Por tener un elevado número de copias se ha encontrado una variabilidad que va desde la ausencia de diferencias entre las copias (25) hasta formas muy divergentes, como es el caso de la cruzipaína 2 
$(26,27)$. El dominio $C$ terminal de la cruzipaína es responsable del carácter inmunodominante antigénico de esta proteína en infecciones humanas naturales (28), en las que la gran mayoría de los anticuerpos detectados en el suero de pacientes chagásicos crónicos está dirigida contra este dominio (29). La cruzipaína y su actividad como proteasa se ha implicado en funciones tan importantes como la supervivencia y la progresión en el ciclo de vida del parásito $(30,31)$, la penetración e invasión del tripomastigote en la célula hospedadora (32-34), la protección contra la respuesta inmune del hospedero (35) y la diseminación del parásito (2).

El análisis de las secuencias disponibles en las bases de datos para estos dos genes muestra un nivel de polimorfismo que no se ha evaluado en cepas de Colombia. Dada la importancia de estos genes en el ciclo de vida del parásito se propuso examinar la variabilidad genética de tripanotión reductasa (TriR) y cruzipaína (Crp) en relación con el origen geográfico y biológico en cepas de $T$. cruzi de Colombia. Conociendo la variabilidad de estos genes en cepas que circulan en nuestro país, se podrá en el futuro proponer estudios de asociación entre esta variabilidad y la enfermedad.

\section{Materiales y métodos}

Se analizaron 36 cepas colombianas de $T$. cruzi y 7 clones de la cepa Cas15, provenientes de diferentes hospederos, vectores y regiones de Colombia (cuadro 1). Se incluyeron las cepas de referencia Tulahuen y CL clasificadas como $T$. cruzi ll. Todas las cepas y los clones provinieron del banco de cepas del Laboratorio de Chagas de la Universidad de Antioquia, donde son mantenidas en medio de cultivo LIT suplementado con suero bovino fetal a $27,5^{\circ} \mathrm{C}(36)$. El ADN del parásito se obtuvo por el método salting out (37) y su concentración se midió por densidad óptica a $260 \mathrm{~nm}$ (38). El ADN se disolvió en $50 \mu \mathrm{lde} \mathrm{H}_{2} \mathrm{O}$ dd y se almacenó a $-20^{\circ} \mathrm{C}$ para su posterior genotipificación.

El diseño experimental incluyo un análisis de las secuencias disponibles en www.ncbi.nlm.nih.gov y www.tigr.org. Para TriR se usaron 41 secuencias de ADN (cuadro 2) y para Crp, 10 secuencias (nueve de Crp1 y una de Crp2, cuadro 3), las cuales fueron alineadas usando el algoritmo Clustal W (39). Para Crp2 se cuenta con mayor número de caracteres y por ello se usó como referencia para el alineamiento. La identificación de sitios polimórficos y su posible discriminación en ensayos de reacción en cadena de la polimerasapolimorfismos de longitud de fragmentos de restricción (polymerase chain reaction-restriction fragment lenght polymorphisms, PCR-RFLP) se realizó usando los programas pDRAW32 (www.acaclone.com), DAMBE (39) y DnaSP (40). Con las secuencias disponibles se diseñaron los cebadores para el fragmento de TriR (TriR directo: 5'AGTGTTACTTTCGAGAGCG3'; TriR inverso: 5'GTAGAAGTCGGATATTTTGG3'), los cuales amplifican una región de $564 \mathrm{pb}$, correspondiente a las posiciones 726 a 1.290 de la secuencia AF359000, pero numeradas en el cuadro 2 como posiciones 1 a 564 según el fragmento amplificado, y para el fragmento del gen Crp (Crp directo: 5'GTTGAGTGCCAGTGGTTTC3'; Crp reverso: 5'TGGTCAGGAGACACTGACC3'), los cuales producen un fragmento de $700 \mathrm{pb}$ correspondiente a las posiciones 1.346 a 2.046 de la secuencia M90067 (cuadro 3).

Las reacciones de amplificación se realizaron en un volumen de $25 \mu \mathrm{L}$ (Tris- $\mathrm{HCl} 10 \mathrm{mM}, \mathrm{pH} 8.0$, $\mathrm{KCl} 50 \mathrm{mM}, \mathrm{MgCL}_{2} 2.4 \mathrm{mM}$, dNTP $0.1 \mathrm{mM}, 0.1 \mu \mathrm{M}$ de cada cebador, $1 \cup$ Taq ADN polimerasa Promega ${ }^{\circledR}$ y 50 ng de ADN molde). El perfil térmico para ambos marcadores fue de un minuto de desnaturalización a $94^{\circ} \mathrm{C}$, un minuto de alineamiento a $54^{\circ} \mathrm{C}$ para $\operatorname{Crp}$ y $56^{\circ} \mathrm{C}$ para $\operatorname{TriR}$ y extensión del cebador por un minuto a $72^{\circ} \mathrm{C}$, para un total de 35 ciclos para Crp y 30 ciclos para TriR. Las reacciones de amplificación se observaron en electroforesis en geles de agarosa al 1,5\% seguida de tinción con bromuro de etidio.

Los fragmentos amplificados incluyen regiones polimórficas que pueden ser resueltas con las enzimas mostradas en las cuadros 2 y 3 . Para cruzipaína, las enzimas Rsa I, Bsu 36l y Ban I permiten la diferenciación de polimorfismos en tercera posición, mientras que Dra III permite la identificación de una transición A:G en la posición 457, la cual da lugar a un cambio de alanina por serina. Ambas formas de la cruzipaína poseen 
Cuadro 1. Origen geográfico, biológico y clasificación previa de los aislamientos de Trypanosoma cruzi. ID se refiere al código de identificación.

\begin{tabular}{|c|c|c|c|c|}
\hline Cepa & Origen geográfico & Origen biológico & ID internacional & Grupo \\
\hline AMP 07 & Antioquia & P. geniculatus & IPANS/CO/1997/AMP07 & I \\
\hline AF 1 & Antioquia & P. geniculatus & I-PANS/CO/93/Af1 & Ilb \\
\hline Yg03 & Antioquia & P. geniculatus & I-PANS/CO/97/YG 03 & 1 \\
\hline Cas 18 & Casanare & D. marsupialis & M.DID/CO/02/CAS18 & I \\
\hline $\mathrm{HA}$ & Casanare & H. sapiens & $\mathrm{M}-\mathrm{HUM} / \mathrm{CO} / 97 / \mathrm{HA}$ & I \\
\hline Cas16 & Casanare & R. prolixus & I.RHO/CO/00/CAS16 & I \\
\hline Cas 15 & Casanare & R. prolixus & TPRX/CO/2000/CAS15 & I \\
\hline B138 & Córdoba & T. dimidiata & I-TRI/CO/99B138 & I \\
\hline B114 & Córdoba & T. dimidiata & I-TRI/CO/99/B114 & I \\
\hline B51 & Córdoba & R. pallescens & TPAC/CO/1999/B51 & I \\
\hline Sb 1 & Córdoba & R. pallescens & $\mathrm{I}-\mathrm{RHO} / \mathrm{CO} / 98 / \mathrm{Sb} 1$ & I \\
\hline Ac17 & Chocó & R. pallescens & I-RHO/CO/99/Ac17 & 1 \\
\hline Mag 11 & Magdalena & R. pallescens & I:RHO/CO/03/MG11 & I \\
\hline Mag 1 & Magdalena & R. pallescens & I.RHO/CO/01/MG1 & 1 \\
\hline Mag5 & Magdalena & E. cuspidatus & TCUS/CO/2003/MG5 & 1 \\
\hline Mag 9 & Magdalena & T. dimidiata & I.TRI/CO/03/MG9 & i \\
\hline Mag8 & Magdalena & T.dimidiata & TDIM/CO/2003/MG8 & i \\
\hline Mag 10 & Magdalena & T.dimidiata & TDIM/CO/2003/MG10 & 1 \\
\hline Putumayo4 & Putumayo & R. robustus & I. RHO/CO/01/PUT-4 & I \\
\hline SN3 & Sierra Nevada & R. prolixus & TPRX/CO/2003/SN3 & I \\
\hline SN 8 & Sierra Nevada & R. prolixus & I. $\mathrm{RHO} / \mathrm{CO} / 03 / \mathrm{SN}-8$ & I \\
\hline SN5 & Sierra Nevada & R. prolixus & TPRX/CO/2003/SN5 & I \\
\hline SN6 & Sierra Nevada & R. prolixus & TPRX/CO/2003/SN6 & I \\
\hline So 6 & Sucre & R. pallescens & I. $\mathrm{RHO} / \mathrm{CO} / 94 / \mathrm{SO} 6$ & I \\
\hline OV 1 & Sucre & P. geniculatus & I-PANS/CO/98/Ov1 & I \\
\hline G20 & Sucre & R. pallescens & TPAC/CO/1997/G20 & I \\
\hline Gal 34 & Sucre & R. pallescens & TPAC/CO/1991/Gal 34 & I \\
\hline W3534 & Sucre & H. sapiens & M-HUM/CO/98/W3534 & I \\
\hline Gal 61 & Sucre & Ratón & MMUS/CO/91/Gal61 & 1 \\
\hline Gal 52 & Sucre & D. marsupialis & MDID/CO/1991/Gal 52 & 1 \\
\hline LB53 & Sucre & T. dimidiata & TDIM/CO/1999/LB53 & I \\
\hline Ov17 & Sucre & P. geniculatus & I.PANS/CO/98/OV17 & 1 \\
\hline So5 & Sucre & R. pallescens & I. RHO/CO/94/SO5 & 1 \\
\hline So8 & Sucre & R. pallescens & TPAC/CO/1995/So8 & I \\
\hline STP 2.2 & Tolima & R. prolixus & I.RHO/CO/91/COY6 & 1 \\
\hline Coy8 & Tolima & D. marsupialis & M.DID/CO/91/COY8 & 1 \\
\hline Tulahuen & Chile & T. infestans & TINF/CH/1956/Tulahuen & II \\
\hline$C L$ & Brasil & T. infestans & TINF/BR/1963/CL & II \\
\hline Cas $15 \mathrm{cl} 47$ & Casanare & R. prolixus & $x$ & 1 \\
\hline Cas $15 \mathrm{cl} 48$ & Casanare & R. prolixus & $x$ & 1 \\
\hline Cas $15 \mathrm{cl} 49$ & Casanare & R. prolixus & $\mathrm{X}$ & 1 \\
\hline Cas $15 \mathrm{cl} 55$ & Casanare & R. prolixus & $\mathrm{X}$ & 1 \\
\hline Cas $15 \mathrm{cl} 56$ & Casanare & R. prolixus & $\mathrm{X}$ & 1 \\
\hline Cas $15 \mathrm{cl} 73$ & Casanare & R. prolixus & $x$ & I \\
\hline Cas $15 \mathrm{cl} 79$ & Casanare & R. prolixus & $x$ & I \\
\hline
\end{tabular}

los tres sitios de corte identificados para la enzima Rsa I. Sin embargo, a diferencia de la Crp1, la Crp2 carece de diana de corte en la posición 142 para la enzima Ban I (cuadro 3). Las enzimas Bsu 36 y Dra III carecen de sitios de corte en esta isoforma (cuadro 3). Las enzimas utilizadas en el análisis de RFLP de tripanotión reductasa - Acy
I, Hae III y Bsm Al - sólo identifican polimorfismos en la tercera posición.

Para verificar la presencia de ambas formas de la cruzipaina se usó la enzima Apa I según el protocolo descrito por Lima et al, 1994 (26); cruzipaína 2 cuenta con un sitio de corte en la posición 298, distinguiendo el sitio variante 297 
Cuadro 2. Posiciones evaluadas y enzimas que las reconocen en las secuencias disponibles en las bases de datos públicas para el gen tripanotión reductasa.

\begin{tabular}{|c|c|c|c|c|c|c|c|}
\hline ID. Secuencia & Grupo & $\begin{array}{c}59^{\star} \\
\text { (HaellI) } \dagger\end{array}$ & $\begin{array}{c}101 \\
(\text { Acy } \mathrm{l} / \mathrm{102})\end{array}$ & $\begin{array}{c}179 \\
(A c y I)\end{array}$ & $\begin{array}{c}211 \\
\text { (Haelll/ 210) }\end{array}$ & $\begin{array}{c}331 \\
\text { (BsmAl) }\end{array}$ & $\begin{array}{c}535 \\
\text { (BsmAl/ 534) }\end{array}$ \\
\hline TC10727 & - & $\mathrm{G}$ & C & A & $\mathrm{G}$ & A & C \\
\hline Z13958 & I & $\mathrm{G}$ & $\mathrm{C}$ & $A$ & $\mathrm{G}$ & A & $\mathrm{C}$ \\
\hline AF358981 & I & $\mathrm{G}$ & $\mathrm{C}$ & $A$ & $\mathrm{G}$ & $A$ & C \\
\hline M38051 & i & $\mathrm{G}$ & $\mathrm{C}$ & $A$ & $\mathrm{~T}$ & A & $\mathrm{C}$ \\
\hline AF358975 & i & $\mathrm{G}$ & $\mathrm{C}$ & $A$ & $\mathrm{G}$ & $A$ & $\mathrm{C}$ \\
\hline AF358982 & I & $\mathrm{G}$ & $\mathrm{C}$ & $A$ & $\mathrm{G}$ & A & C \\
\hline AF358987 & i & $\mathrm{G}$ & $\mathrm{C}$ & $A$ & $\mathrm{G}$ & $A$ & $\mathrm{C}$ \\
\hline AF358971 & i & $\mathrm{G}$ & $\mathrm{C}$ & $A$ & $\mathrm{G}$ & $A$ & $\mathrm{C}$ \\
\hline AF358973 & I & $\mathrm{G}$ & $\mathrm{C}$ & $A$ & $\mathrm{G}$ & $A$ & $\mathrm{C}$ \\
\hline $\begin{array}{l}\text { AF358972, AF35976 } \\
\text { AF358985,AF358984, }\end{array}$ & i & $\mathrm{G}$ & C & A & $\mathrm{G}$ & A & C \\
\hline $\begin{array}{l}\text { AF358983,AF358977, } \\
\text { AF358979,AF358980 }\end{array}$ & I & $G$ & C & $A$ & $G$ & A & C \\
\hline $\begin{array}{l}\text { AF358978,AF358970, } \\
\text { AF358969,AF358974 }\end{array}$ & I & $\mathrm{G}$ & C & $A$ & G & A & C \\
\hline AF358989, AF358988 & II & $\mathrm{G}$ & $\mathrm{T}$ & A & $\mathrm{G}$ & A & C \\
\hline AF358986 & II & $\mathrm{G}$ & $\mathrm{C}$ & $A$ & $\mathrm{G}$ & $A$ & $A$ \\
\hline M97953 & II & $\mathrm{G}$ & $\mathrm{C}$ & A & $\mathrm{G}$ & $A$ & $\mathrm{C}$ \\
\hline $\begin{array}{l}\text { AF359003,AF358997, } \\
\text { AF358994, AF358993, } \\
\text { AF358992,AF359001, } \\
\text { AF358999,AF359005 }\end{array}$ & II & $\mathrm{G}$ & C & A & $\mathrm{T}$ & A & C \\
\hline $\begin{array}{l}\text { AF359002,AF358996, } \\
\text { AF359000,AF358998, } \\
\text { AF359004,AF358991 }\end{array}$ & II & $G$ & $\mathrm{~T}$ & A & $\mathrm{G}$ & A & $\mathrm{C}$ \\
\hline AF358990 & ॥ & $\mathrm{G}$ & $\mathrm{T}$ & A & $\mathrm{G}$ & A & C \\
\hline AF358995 & II & $\mathrm{G}$ & C & A & $\mathrm{T}$ & A & $\mathrm{C}$ \\
\hline
\end{tabular}

* Posición en la cual la enzima realiza el corte.

† Entre paréntesis se indica la enzima que realiza el corte y la posición del polimorfismo que identifica.

(cuadro 3) y generando fragmentos de 297 y 403 $\mathrm{pb}$.

Los productos de amplificación se sometieron a digestiones simples a un volumen final de $20 \mu \mathrm{l}$ con $1 \mathrm{U}$ de enzima a $37^{\circ} \mathrm{C}$ por 16 horas. Una alícuota de $12 \mu \mathrm{L}$ se analizó en geles de agarosa al $3 \%$ teñidos con bromuro de etidio. El resultado de la digestión fue registrado en un analizador de imágenes Gel Doc (BioRad, USA). Los patrones de bandas obtenidos fueron analizados con el software Imagej 1.33 (http://rsb.info.nih.gov/ij) y Quantity One (BioRad, USA). Para cada cepa y para cada gen se definieron los genotipos de acuerdo con los patrones observados en los electroferogramas.

\section{Resultados}

Los productos de amplificación obtenidos en los ensayos de PCR para TrR y Crp se muestran en la figura 1. Algunos de los perfiles de las digestiones simples se muestran en las figuras 2 a 6 .

Los resultados de las digestiones se representan como genotipos compuestos de las digestiones simples. De seis sitios evaluados para TriR, sólo dos mostraron polimorfismo. Sólo la combinación de los genotipos obtenidos para las enzimas Hae III y Acy I muestran patrones polimórficos, denominados TriR 1, 2 y 3 (cuadro 4). El genotipo TriR 3 puede definirse con certeza debido a que el corte sólo se presenta ante la presencia de los alelos C y G para los sitios Acy I-102 y Hae III- 
Cuadro 3. Posiciones evaluadas y enzimas que las reconocen en las secuencias disponibles en las bases de datos públicas para el gen cruzipaína.

\begin{tabular}{|c|c|c|c|c|c|c|c|c|c|c|}
\hline Secuencia & Grupo & $\begin{array}{c}142^{*} \\
(B a n l / \\
147) \dagger\end{array}$ & $\begin{array}{c}151 \\
\text { (Rsal) }\end{array}$ & $\begin{array}{c}222 \\
\text { (DrallI/ } \\
225)\end{array}$ & $\begin{array}{c}226 \\
(\text { Banl/ } \\
231)\end{array}$ & $\begin{array}{c}257 \\
(B s u 36 \mathrm{l} / \\
258)\end{array}$ & $\begin{array}{c}299 \\
(\text { Apal/ } \\
297)\end{array}$ & $\begin{array}{c}430 \\
(R s a l / \\
432)\end{array}$ & $\begin{array}{c}461 \\
\text { (DrallI/ } \\
462)\end{array}$ & $\begin{array}{r}613 \\
(R s a l \\
612)\end{array}$ \\
\hline M84342 & II & C & $\mathrm{T}$ & $\mathrm{G}$ & $\mathrm{C}$ & $\mathrm{T}$ & $\mathrm{T}$ & C & G & $G$ \\
\hline TC10685 & I & $\mathrm{C}$ & $\mathrm{T}$ & $\mathrm{G}$ & $\mathrm{C}$ & $\mathrm{G}$ & $\mathrm{T}$ & C & $\mathrm{G}$ & $\mathrm{G}$ \\
\hline AF314930 & II & $\mathrm{C}$ & $\mathrm{T}$ & $\mathrm{G}$ & $\mathrm{C}$ & $\mathrm{T}$ & $\mathrm{T}$ & $\mathrm{C}$ & $\mathrm{G}$ & $\mathrm{C}$ \\
\hline AF314929 & II & $\mathrm{C}$ & $\mathrm{T}$ & $\mathrm{G}$ & $\mathrm{C}$ & $\mathrm{T}$ & $\mathrm{T}$ & $\mathrm{C}$ & $\mathrm{G}$ & $\mathrm{G}$ \\
\hline AY0317 & II & $\mathrm{C}$ & $\mathrm{T}$ & $\mathrm{G}$ & $\mathrm{C}$ & $\mathrm{T}$ & $\mathrm{T}$ & $\mathrm{T}$ & $\mathrm{G}$ & $\mathrm{G}$ \\
\hline AF265227 & I & $\mathrm{C}$ & $\mathrm{T}$ & $\mathrm{G}$ & $\mathrm{T}$ & $\mathrm{G}$ & $\mathrm{T}$ & $\mathrm{C}$ & $\mathrm{T}$ & $\mathrm{G}$ \\
\hline AF265226 & I & $\mathrm{C}$ & $\mathrm{T}$ & $\mathrm{G}$ & $\mathrm{C}$ & $\mathrm{G}$ & $\mathrm{T}$ & $\mathrm{C}$ & $G$ & $\mathrm{G}$ \\
\hline U41454 & i & $\mathrm{C}$ & $\mathrm{T}$ & $\mathrm{G}$ & $\mathrm{C}$ & $\mathrm{G}$ & $\mathrm{T}$ & $\mathrm{C}$ & $G$ & $\mathrm{G}$ \\
\hline AF004594 & II & C & $\mathrm{T}$ & $\mathrm{T}$ & $\mathrm{C}$ & $\mathrm{T}$ & $\mathrm{T}$ & $\mathrm{C}$ & $\mathrm{G}$ & $\mathrm{G}$ \\
\hline M90067 & & & & & & & & & & \\
\hline (Cruzipaína2) & I & $\mathrm{T}$ & $\mathrm{T}$ & $\mathrm{G}$ & C & $G$ & $G \ddagger$ & C & $\mathrm{T}$ & $\mathrm{G}$ \\
\hline
\end{tabular}

* Posición en la cual la enzima realiza el corte.

† Entre paréntesis se indica la enzima que realiza el corte y la posición del polimorfismo que identifica.

‡ Sitio para distinguir las copias reportadas de cruzipaína.

210 , respectivamente; este genotipo, presente en 35 de 38 cepas, se encuentra en las cepas caracterizadas como T. cruzi I, en las cuales es definido por la ausencia del alelo $T$ en ambas posiciones (cuadro 4). El genotipo TriR 1, heterocigótico para ambas posiciones, se



Figura 1. Productos de amplificación obtenidos para cruzipaína (izquierda) y tripanotión reductasa (derecha). Sólo se muestra el producto para algunas cepas del grupo I (Cas15) y grupo II (AF1, Tulahuen y CL). MP = marcador de peso de $100 \mathrm{pb}$. encontró en las dos cepas de referencia, de acuerdo con lo reportado en las bases de datos (cuadro 2, identificaciones de acceso: AF358997 y AF359002 para Tulahuen y AF358999 y AF358998 para CL), mientras que el genotipo TriR 2 se encontró en la cepa AF1 proveniente de Amalfi, Antioquia (cuadro 4). Para estos dos genotipos se infiere el alelo $T$ en ambas posiciones debido a que es el polimorfismo alternativo presente en las secuencias analizadas y a que no se reporta otro sitio polimórfico alrededor de la diana de reconocimiento de la enzima. La distribución de estos genotipos no mostró ninguna asociación con origen geográfico o biológico (datos no mostrados).

En los ensayos de digestión para Crp, la enzima Apa I permitió identificar la cruzipaína 2 diferenciada por el polimorfismo en la posición 297 (figura 3). La presencia de por lo menos dos copias resulta en patrones de digestión complejos, en los que es posible obtener más de dos alelos para cada posición; para facilitar el análisis, denominamos como genotipo "heterocigoto" a aquel que poseía más de un alelo en un locus, lo cual puede corresponder a diferencias entre copias de Crp o a una condición heterocigótica en sentido estricto.

Basados en las secuencias reportadas para Crp, los sitios 150 a 153 representan una diana de 


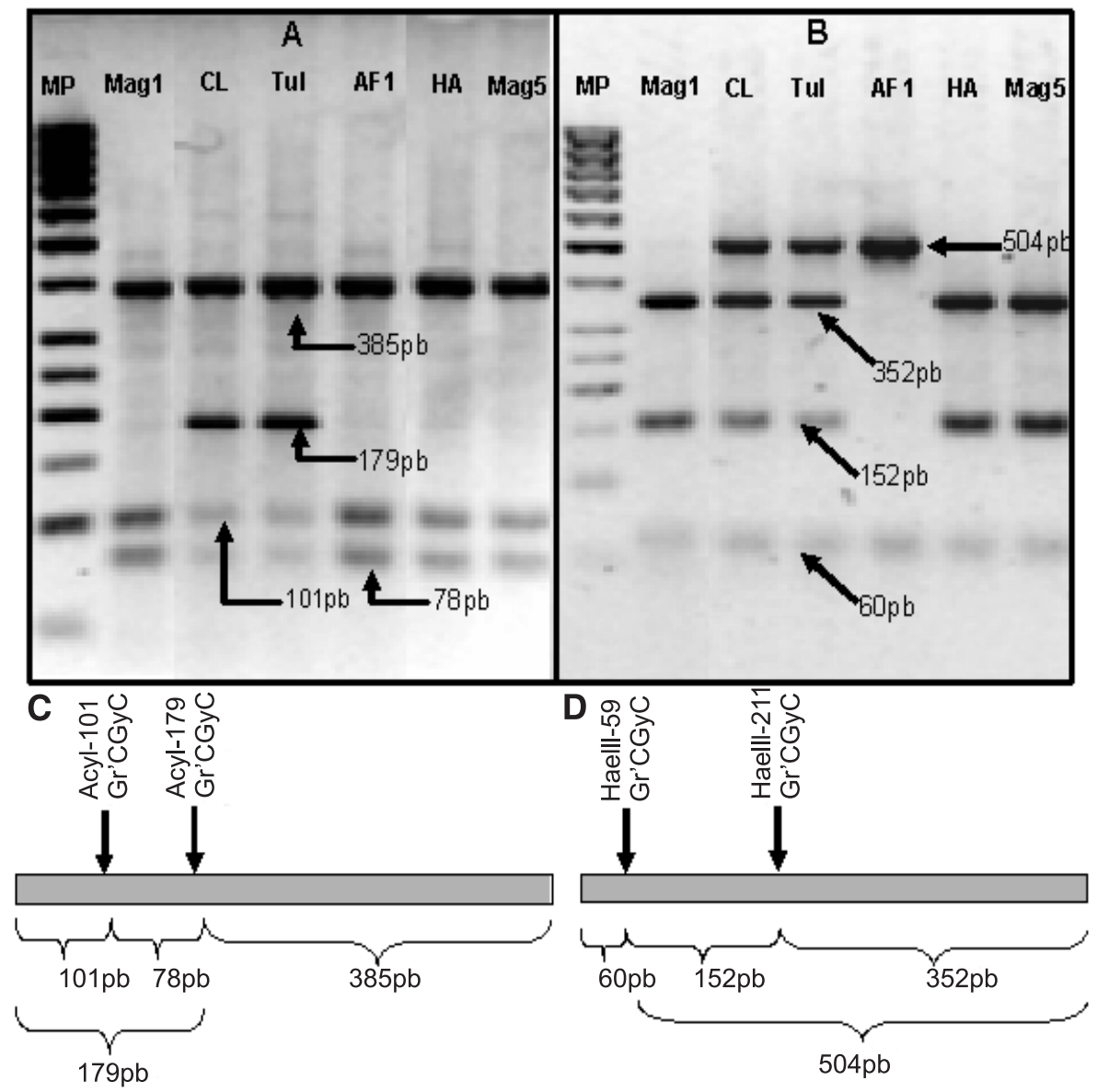

Figura 2. Patrones RFLP para TriR con la enzima Acy I (A) y Hae III (B) en algunas cepas de los grupos I (Mag1, HA y Mag5) y grupo II (CL, Tulahuen y AF1). MP = Marcador de peso de $50 \mathrm{pb}$. Los tamaños de los fragmentos se muestran en los electroferogramas ( $A$ y B) y en los mapa de restricción ( $C$ y $D$ ), para cada una de las enzimas y su diana de reconocimiento ( $y=$ Purina; $r=$ Pirimidina). Sólo los perfiles para las enzimas que produjeron patrones polimórficos entre cepas son mostrados.

Cuadro 4. Genotipos compuestos Tripanotión reductasa.

\begin{tabular}{lccc}
\hline Cepa & $\begin{array}{c}\text { Acy I } \\
\mathbf{1 0 2}\end{array}$ & $\begin{array}{c}\text { Hae III } \\
\mathbf{2 1 0}\end{array}$ & Genotipo \\
\hline TUL, CL & C/T* & G/T† & TriR 1 \\
AF1 & C/C & T/T & \\
HA, Cas18, Mag5, SN5, SN8, OV1, Cas16, Mag10, LB53, & & & TriR 3 \\
Cas15, Amp07, Yg03, B138, B114, B51, Sb1, Ac17, Mag1, & C/C & G/G & \\
Mag8, Mag9, Mag11, Putumay04, SN3, SN6, So6, G20, Gal34 & & & \\
W3534, Gal61, Gal52, Ov17, So5, So8, STP2.2, Coy8, & & & \\
Clones Cas 15 & & & \\
\hline
\end{tabular}

${ }^{*} \mathrm{C}$ corte en la posición 101 con la enzima Acy I †G corte en la posición 211 con la enzima Hae III 


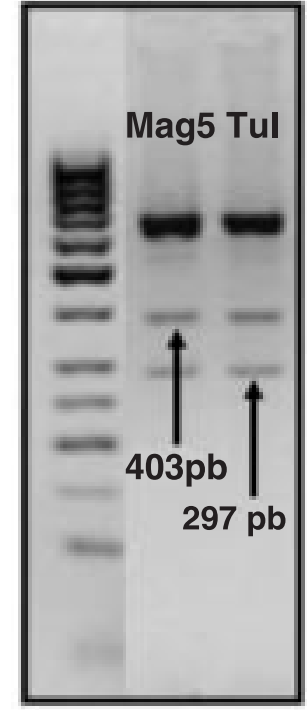

Figura 3. Digestión simple de cruzipaína con la enzima Apa I en cepas del grupo I (Mag5) y del grupo II (Tulahuen) para distinguir la presencia de más de una copia del gen usando el protocolo descrito por Lima et al., 1994.

reconocimiento para la enzima $R s a$ I con corte en la posición 151 (cuadro 3); estos sitios se reportan monomórficos en todas las secuencias (datos no mostrados), pero en algunas cepas analizadas en este estudio dicha diana se perdió originando genotipos heterocigotos; sus alelos se representan como $1 / 0$ debido a que no se puede comprobar cuál es el sitio polimórfico dentro de la diana de reconocimiento de la enzima (cuadro 5). Los alelos Ban I-C y Rsa I-C en las posiciones 231 y 432 , respectivamente, se definen con certeza porque la enzima sólo corta cuando éstos están presentes. Los alelos Ban I-T, Bsu 36I-G y Rsa I$T$ en las posiciones 231, 258 y 432 se infieren debido a que es el polimorfismo alternativo reportado en las secuencias analizadas y a que no se reporta otro polimorfismo alrededor de la diana de reconocimiento para cada enzima. Los sitios evaluados con la enzima Dra III fueron monomórficos y por esto no se consideran en los genotipos compuestos.

A diferencia de TriR, y probablemente por la presencia de más de una copia, para Crp se obtuvieron seis genotipos compuestos representados como Crp 1 a Crp 6 (cuadro 5). El genotipo Crp 5, homocigótico para todos los loci, agrupa

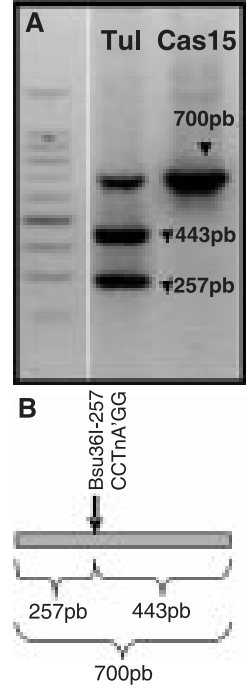

Figura 4. Patrones RFLP para Crp con la enzima Bsu 36l en cepas del grupo I (Cas15) y II (Tulahuen). Los tamaños de los fragmentos se muestran en el electroferograma $(A)$ y en el mapa de restricción (B).

26 cepas clasificadas como T. cruzi I (cuadro 5). El genotipo Crp 1 se encontró en las cepas del grupo $T$. cruzill y es diferenciado por la presencia del alelo T en la posición 258 (cuadro 5). Éste y el genotipo Crp 3 son los más polimórficos; los genotipos Crp 2, Crp 4 y Crp 6 mostraron polimorfismo en la diana alrededor del sitio 151; adicionalmente, Crp 2 varió en la posición 432, y Crp 4 y Crp 6 variaron en la posición 231 (cuadro 5).

Para Crpfue posible establecer algunas asociaciones entre los genotipos y el origen geográfico y biológico de las cepas (cuadro 6). Cuando se consideró el origen geográfico, la región del oriente (departamento de Casanare) mostró ser más diversa, con cuatro de los seis genotipos; dos de ellos, Crp 3 y Crp 6, son específicos de esta región y son diferenciados por el alelo T en el sitio 231. El genotipo Crp2 se encuentra en el noroccidente (Sucre, Magdalena, Sierra Nevada) y oriente de Colombia (Casanare); este genotipo también se encontró en aislamientos de Rhodnius prolixus, Pastrongylus geniculatus y Eratyrus cuspidatus; entre los hospedadores mamíferos este genotipo sólo se encontró en Didelphis marsupialis (cuadro 6). El genotipo Crp 4 tiene una distribución similar al genotipo Crp 2, pero está ausente en la Sierra 
Cuadro 5. Genotipos compuestos de cruzipaína.

\begin{tabular}{|c|c|c|c|c|c|}
\hline Cepa & $\begin{array}{c}\text { Rsa I } \\
151\end{array}$ & $\begin{array}{l}\text { Ban I } \\
231^{*}\end{array}$ & $\begin{array}{c}\text { Bsu 36I } \\
258 \dagger\end{array}$ & $\begin{array}{l}\text { Rsa I } \\
432 \ddagger\end{array}$ & Genotipo \\
\hline CL, Tulahuen, AF1 & $1 / 0 \S$ & $\mathrm{C} / \mathrm{C}$ & $\mathrm{G} / \mathrm{T}$ & $\mathrm{C} / \mathrm{T}$ & Crp 1 \\
\hline Cas18,Mag5, SN5,SN8,OV 1 & $1 / 0$ & $\mathrm{C} / \mathrm{C}$ & $\mathrm{G} / \mathrm{G}$ & $\mathrm{C} / \mathrm{T}$ & Crp 2 \\
\hline $\mathrm{HA}$ & $1 / 0$ & $\mathrm{C} / \mathrm{T}$ & $\mathrm{G} / \mathrm{G}$ & $\mathrm{C} / \mathrm{T}$ & Crp 3 \\
\hline Cas16, Mag10,LB53 & $1 / 0$ & $\mathrm{C} / \mathrm{C}$ & $\mathrm{G} / \mathrm{G}$ & $\mathrm{C} / \mathrm{C}$ & Crp 4 \\
\hline $\begin{array}{l}\text { Amp07, Yg03, B138, B114, B51, Sb1, Ac17, Mag1, } \\
\text { Mag8, Mag9, Mag11, Putumayo4, SN3, SN6, So6, } \\
\text { G20, Gal34, W3534, Gal61, Gal52, Ov17, So5, } \\
\text { So8, STP2.2,Coy8, }\end{array}$ & $1 / 1$ & $\mathrm{C} / \mathrm{C}$ & $\mathrm{G} / \mathrm{G}$ & $\mathrm{C} / \mathrm{C}$ & Crp 5 \\
\hline Cas 15, Clones Cas 15 & $1 / 1$ & $\mathrm{C} / \mathrm{T}$ & $\mathrm{G} / \mathrm{G}$ & $\mathrm{C} / \mathrm{C}$ & Crp6 \\
\hline
\end{tabular}

'C corte en la posición 226

†G no corte en la posición 257

$\ddagger C$ corte en la posición 430

$\S 1=$ corte 0 = no corte

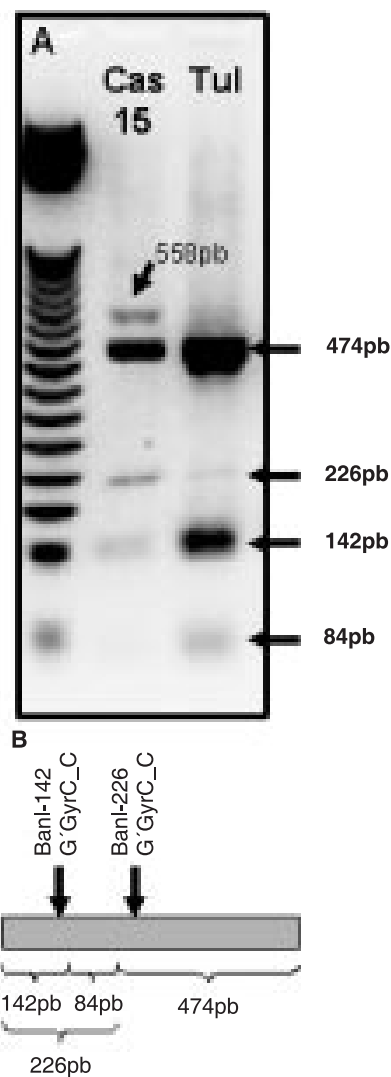

Figura 5. Patrones RFLP para Crp con la enzima Ban I en cepas del grupo I (Cas15) y II (Tulahuen). Los tamaños de los fragmentos se muestran en el electroferograma (A) y en el mapa de restricción (B). La banda de 558 pb se explica por la ausencia de corte en la posición 226 y la banda de 226 pb, por ausencia de corte en la posición 142.

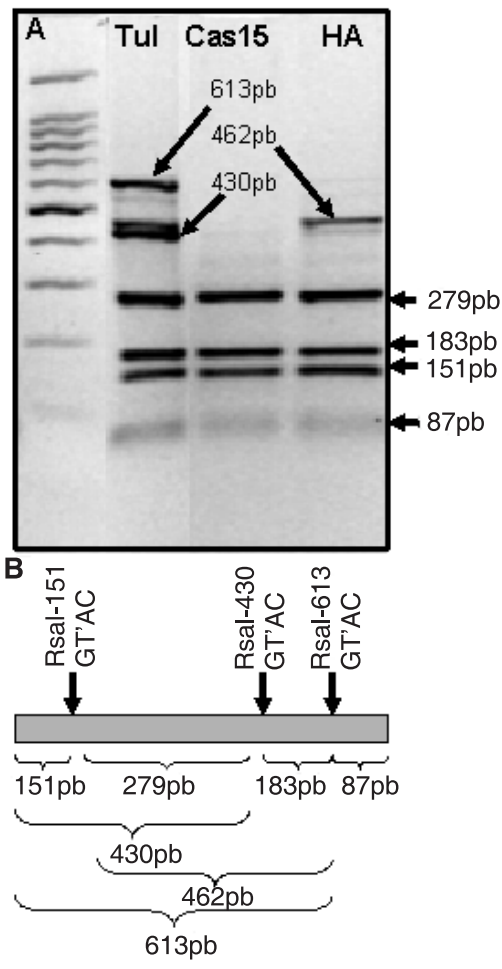

Figura 6. Patrones RFLP para Crp con la enzima Rsal en cepas del grupo I (Cas15 y HA) y II (Tulahuen). Los tamaños de los fragmentos se muestran en el electroferograma $(A)$ y en el mapa de restricción (B). La banda de $430 \mathrm{pb}$ se explica por la ausencia de corte en la posición 151; la banda de 462 pb, por la ausencia de corte en la posición 430; la banda de $613 \mathrm{pb}$, por la ausencia simultánea de corte en las posiciones 151 y 430. 
Cuadro 6. Distribución de genotipos compuestos de acuerdo con el departamento o región de procedencia y con el origen biológico de las cepas estudiadas.

\begin{tabular}{|c|c|c|}
\hline Genotipo compuesto & $\begin{array}{l}\text { Departamento/Región } \\
\text { (No. de cepas) }\end{array}$ & $\begin{array}{l}\text { Origen biológico } \\
\text { (No. de cepas) }\end{array}$ \\
\hline $\begin{array}{l}\text { Crp } 1 \\
* 1 / 0 \mathrm{C} / \mathrm{C} \mathrm{G} / \mathrm{T} \mathrm{C} / \mathrm{T}\end{array}$ & $\begin{array}{l}\text { Chile (1) } \\
\text { Brasil (1) } \\
\text { Antioquia (1) }\end{array}$ & $\begin{array}{l}\text { T. infestans (1) } \\
\text { T. infestans (1) } \\
\text { P. geniculatus (1) }\end{array}$ \\
\hline $\begin{array}{l}\text { Crp } 2 \\
1 / 0 \mathrm{C} / \mathrm{C} \mathrm{G} / \mathrm{G} \mathrm{C} / \mathrm{T}\end{array}$ & $\begin{array}{l}\text { Casanare (1) } \\
\text { Magdalena(1) } \\
\text { Sierra Nevada (2) } \\
\text { Sucre (1) }\end{array}$ & $\begin{array}{l}\text { D. marsupialis (1) } \\
\text { E. cuspidatus (1) } \\
\text { R. prolixus (2) } \\
\text { P. geniculatus (1) }\end{array}$ \\
\hline $\begin{array}{l}\text { Crp } 3 \\
1 / 0 \mathrm{C} / \mathrm{T} \mathrm{G} / \mathrm{G} \mathrm{C} / \mathrm{T}\end{array}$ & Casanare (1) & H. sapiens (1) \\
\hline $\begin{array}{l}\text { Crp } 4 \\
1 / 0 \mathrm{C} / \mathrm{C} \mathrm{G} / \mathrm{G} \mathrm{C} / \mathrm{C}\end{array}$ & $\begin{array}{l}\text { Casanare (1) } \\
\text { Magdalena (1) } \\
\text { Sucre (1) }\end{array}$ & $\begin{array}{l}\text { R. prolixus (1) } \\
\text { T. dimidiata (1) } \\
\text { T. dimidiata (1) }\end{array}$ \\
\hline & $\begin{array}{l}\text { Antioquia (2) } \\
\text { Córdoba (4) } \\
\text { Chocó (1) }\end{array}$ & $\begin{array}{l}\text { P. geniculatus (2) } \\
\text { T. dimidiata (2) } \\
\text { R. pallescens (2) } \\
\text { R. pallescens (1) }\end{array}$ \\
\hline $\begin{array}{l}\text { Crp } 5 \\
1 / 1 \mathrm{C} / \mathrm{C} \mathrm{G} / \mathrm{G} \mathrm{C} / \mathrm{C}\end{array}$ & Magadalena (4) & $\begin{array}{l}\text { R. pallescens (2) } \\
\text { T. dimidiata (2) }\end{array}$ \\
\hline & $\begin{array}{l}\text { Sierra Nevada (2) } \\
\text { Putumayo (1) } \\
\text { Sucre (9) }\end{array}$ & $\begin{array}{l}\text { R. prolixus (2) } \\
R . \text { robustus (1) } \\
R . \text { pallescens (5) } \\
P . \text { geniculatus (1) } \\
D . \text { maruspialis (1) } \\
\text { H. sapiens (1) } \\
\text { Ratón (1) }\end{array}$ \\
\hline & Tolima (2) & $\begin{array}{l}\text { R. prolixus (1) } \\
\text { D. marsupialis (1) }\end{array}$ \\
\hline $\begin{array}{l}\text { Crp } 6 \\
1 / 1 C / T \text { G/G } C / C\end{array}$ & Casanare (1) & R. prolixus (1) \\
\hline
\end{tabular}

*1 $=$ corte; 0 = no corte

Nevada de Santa Marta; este genotipo está asociado a $R$. prolixus y Triatoma dimidiata, principales vectores domiciliados.

El genotipo Crp 5 fue el más frecuente; se presentó en todas las regiones del occidente de la cordillera oriental, en la totalidad de los hospederos mamíferos y en la mayoría de los insectos vectores, a excepción de E. cuspidatus; es el único genotipo encontrado en los 10 aislamientos de Rhodnius pallescens. En $R$. prolixus se encontró el mayor número de genotipos (Crp 2, Crp 4, Crp 5 y Crp 6).

\section{Discusión}

La evaluación de TriR con las enzimas Acy I y Hae III permitió distinguir cepas de los grupos previamente descritos con otros marcadores moleculares. Las cepas del grupo T. cruzi I evaluadas en este estudio mostraron en las posiciones 102 y 211 ausencia del alelo T (cuadro 4). Al considerar las secuencias reportadas, el alelo T en la posición 102 está ausente de todas las cepas del grupo I, como también en algunas del grupo II; el alelo T en la posición 211 fue consistente con las cepas del grupo T. cruzill en nuestros ensayos; sin embargo, los datos de secuencias muestran que este alelo también puede estar presente en cepas de $T$. cruzi I (cuadro 2), por lo que no puede considerársele un alelo específico de grupo.

El gen TriR es codificado por un locus único en el genoma de $T$. cruzi (18) y la condición 
heterocigótica observada en el genotipo compuesto TriR 1 (C/T y G/T) podría reflejar cualquiera de varias situaciones: una combinación de clones en la cepa (41-43), o una heterocigosis "real" causada por mutación o intercambio genético dentro del grupo $T$. cruzi II (18,44-47). Si se considera la naturaleza heterogénea de los aislamientos originales de cepas de $T$. cruzi (42), ello podría argumentarse a favor de la primera situación. Sin embargo, no es posible descartar que los genotipos heterocigotos encontrados representen eventos antiguos de intercambio génico (18,44-47), en particular en el sitio 210, el cual puede ser producto de intercambio entre genotipos homocigotos.

La evaluación del gen TriR en cepas de Colombia mostró en nuestro análisis una baja diversidad de genotipos. El cálculo del polimorfismo $(\theta)$ y diversidad nucleotídica $(\pi)$ a partir de las secuencias reportadas para este gen fue de $0,0083+/-0,00186$ y $0,011+/-0,00062$, respectivamente. Una relación de menor polimorfismo con una mayor diversidad permite suponer que se trata de un gen sujeto a selección, o que efectivamente existe una estructura clonal en las cepas de Colombia; sin embargo, esta afirmación requeriría de confirmación, dado que los rangos de estos valores se superponen. Esta afirmación puede contradecirse si se considera que el polimorfismo en dichas secuencias se localiza en terceras posiciones del codón, dando lugar a cambios neutros. La tripanotión reductasa cumple una función en la detoxificación de productos endógenos y exógenos en el ciclo de vida del parásito y nuestros resultados no muestran una asociación con origen biológico; ello sugiere que, de estar sujeta a selección, su fuerza no ha operado diferencialmente entre los distintos orígenes biológicos y geográficos de donde proceden las cepas. Por este bajo polimorfismo, esta proteína podría constituirse en un blanco adecuado para diseños terapéuticos $(48,49)$.

La presencia de por lo menos dos formas de Crp hace más complejo el análisis tanto de los genotipos como de su asociación con orígenes geográficos y biológicos. El mayor polimorfismo de este gen (cuatro sitios polimórficos de ocho sitios evaluados, excluido el sitio Apa I) dio lugar a seis genotipos, en contraste con tres genotipos para TriR, lo cual concuerda con la presencia de más de una copia del gen. Desde este punto de vista, muchas cepas (genotipo Crp 5) podrían representar eventos de concertación génica (5052) en los que los loci de ambas copias son homocigóticos; pero esto requiere asumir hipótesis ad hoc de mutación para los mismos loci en otras cepas (genotipos Crp 1 a 4).

Algunos estudios previos con cepas de Colombia han podido establecer relaciones entre genotipos de $T$. cruzi con ciclos de transmisión $(53,54)$. En este estudio no fue posible establecer relaciones entre genotipos TriRy Crpy ciclos de transmisión, debido a que no se evaluaron cepas aisladas de vectores domiciliados; las cepas $\mathrm{SN}$ se obtuvieron de $R$. prolixus al interior de las viviendas, pero su ubicación dentro de un nicho selvático en la Sierra Nevada de Santa Marta podría introducir errores en dichas comparaciones. Sin embargo, se pudo evaluar la relación entre genotipos y el origen geográfico y biológico de las cepas, en particular con las especies de vectores.

En cuanto a Crp, se puede concluir que el departamento del Casanare es la región con más diversidad de genotipos (cuadro 6). De acuerdo con esto, en estudios previos $(53,55)$ se determinó que la región de los Llanos Orientales exhibió un alto número de fenotipos isoenzimáticos. Dada la alta prevalencia de la enfermedad en esta región (IV Reunión de la Comisión Intergubernamental de la Iniciativa Andina de Control de la Transmisión Vectorial y Transfusional de Chagas. Guayaquil, 2003;56,57), valdría la pena formular hipótesis sobre la diversidad de $T$. cruzien relación con la eficacia biológica (58) y con la epidemiología de la enfermedad.

En cuanto a las asociaciones con los vectores, también se puede concluir que en $R$. pallescens, el vector/hospedero de donde proviene el mayor número de cepas en este estudio, se encontró exclusivamente el genotipo compuesto Crp 5; vale la pena evaluar también una hipótesis de especificad parásito-vector ampliando los marcadores en aislamientos de este vector. En contraste, en las demás especies de vectores se encontró más de un genotipo; en $R$. prolixus, 
principal vector de la enfermedad de Chagas en el país, se encontró el mayor número de genotipos; éste es un insecto ampliamente distribuido en regiones distantes de Colombia; la importancia de este aspecto también puede evaluarse en relación con sus implicaciones por sus hábitos antropofílicos y su domiciliación. Las cepas provenientes de $P$. geniculatus, aunque con una distribución más restringida, también mostraron tres de seis genotipos; esta diferencia en la diversidad genotípica entre especies podría reflejar el efecto de filtros biológicos selectivamente diferentes $(59,60)$.

Usando marcadores de isoenzimas, RAPDs y ADNr, se ha podido demostrar la mayor prevalencia del grupo $T$. cruzil en el país $(53-55,61)$. Con dos sistemas diferentes en genes codificadores de proteínas también fue posible en este estudio reproducir esta observación, agrupándose la mayoría de cepas del grupo I dentro de los genotipos TriR 3 y Crp 5; esta consistencia de agrupación con diferentes sistemas refuerza así la estructura clonal de las poblaciones de $T$. cruzi.

Los resultados de este estudio sugieren que estos genes son útiles como posibles marcadores moleculares para determinar y diferenciar variedades genéticas en $T$. cruzi, y permiten plantear la futura evaluación de la hipótesis sobre la interacción parásito-hospedero.

\section{Conflicto de intereses}

Los autores declaramos que no existe ningún conflicto de intereses.

\section{Financiación}

El estudio fue desarrollado como parte del proyecto 1115-04-14387 financiado por Conciencias.

\section{Referencias}

1. Tibayrenc $\mathbf{M}$, Kjellberg F, Ayala FJ. A clonal theory of parasitic protozoa: the population structures of Entamoeba, Giardia, Leishmania, Naegleria, Plasmodium, Trichomonas and Trypanosoma and their medical and taxonomical consequences. Proc Natl Acad Sci USA 1990;87:2414-8.

2. Scharfstein J, Morrot A. A role for extracellular amastigotes in the immunopathology of Chagas disease. Mem Inst Oswaldo Cruz 1999;94(Suppl 1):51-63.
3. Brener Z. Biology of Trypanosoma cruzi. Annu Rev Microbiol 1973;27:347-82.

4. Melo RC, Brener Z. Tissue tropism of different Trypanosoma cruzi strains. J Parasitol 1978;64:47582.

5. Andrade Z, Brener ZE. Trypanosoma cruzi e Doença de Chagas. Rio de Janeiro: Ed. Guanabar Koogan; 1979.

6. Andrade SG, Andrade V, Brodskyn C, Magalhaes JB, Netto MB. Immunological response of Swiss mice to infection with three different strains of Trypanosoma cruzi. Ann Trop Med Parasitol 1985;79:397-407.

7. de Castro SL, de Meirelles M de N. Effect of drugs on Trypanosoma cruzi and on its interaction with heart muscle cell in vitro. Mem Inst Oswaldo Cruz 1987;82:209-18.

8. Neal RA, van Bueren J. Comparative studies of drug susceptibility of five strains of Trypanosoma cruzi in vivo and in vitro. Trans $\mathrm{R}$ Soc Trop Med Hyg 1988;82:709-14.

9. Vago AR, Andrade LO, Leite AA, d'Avila Reis D, Macedo AM, Adad SJ, et al. Genetic characterization of Trypanosoma cruzi directly from tissues of patients with chronic Chagas disease: differential distribution of genetic types into diverse organs. Am J Pathol 2000;156:1805-9.

10. Morel C, Chiari E, Camargo EP, Mattei DM, Romanha AJ, Simpson L. Strains and clones of Trypanosoma cruzi can be characterized by pattern of restriction endonuclease products of kinetoplast DNA minicircles. Proc Natl Acad Sci USA 1980;77:6810-4.

11. Souto RP, Zingales B. Sensitive detection and strain classification of Trypanosoma cruzi by amplification of a ribosomal RNA sequence. Mol Biochem Parasitol 1993;62:45-52.

12. Tibayrenc M, Neubauer K, Barnabe C, Guerrini F, Skarecky D, Ayala FJ. Genetic characterization of six parasitic protozoa: parity between random-primer DNA typing and multilocus enzyme electrophoresis. Proc Natl Acad Sci USA 1993; 90:1335-9.

13. Souto RP, Fernandes O, Macedo AM, Campbell DA, Zingales B. DNA markers define two major phylogenetic lineages of Trypanosoma cruzi. Mol Biochem Parasitol 1996;83:141-52.

14. Momen H. Taxonomy of Trypanosoma cruzi: a commentary on characterization and nomenclature. Mem Inst Oswaldo Cruz 1999;94(Suppl. 1):181-4.

15. Brisse S, Dujardin JC, Tibayrenc M. Identification of six Trypanosoma cruzi lineages by sequencecharacterized amplified region markers. Mol Biochem Parasitol 2000;111:95-105.

16. Brisse S, Barnabé C, Tibayrenc M. Trypanosoma cruzi: How many relevant phylogenetic subdivisions are there? Parasitol Today 1998;14:178-9. 
17. El-Sayed NM, Myler PJ, Bartholomeu DC, Nilsson D, Aggarwal G, Tran A, et al. The genome sequence of Trypanosoma cruzi, etiologic agent of Chagas disease. Science 2005;309:409-15.

18. Machado CA, Ayala FJ. Nucleotide sequences provide evidence of genetic exchange among distantly related lineages of Trypanosoma cruzi. Proc Natl Acad Sci USA 2001;98:7396-401.

19. Krieger S, Schwarz W, Ariyanayagam MR, Fairlamb AH, Krauth-Siegel RL, Clayton C. Trypanosomes lacking trypanothione reductase are avirulent and show increased sensitivity to oxidative stress. Mol Microbiol 2000;35:542-52.

20.Schmidt A, Krauth-Siegel RL. Enzymes of the trypanothione metabolism as targets for antitrypanosomal drug development. Curr Top Med Chem 2002;11:1239-59.

21. Fairlamb AH, Cerami A. Metabolism and functions of trypanothione in the Kinetoplastida. Annu Rev Microbiol 1992;46:695-729.

22. Dumas C, Ouellette M, Tovar J, Cunningham ML, Fairlamb AH, Tamar S, et al. Disruption of the trypanothione reductase gene of Leishmania decreases its ability to survive oxidative stress in macrophages. EMBO J 1997;16:2590-8.

23. Eakin AE, Mills AA, Harth G, McKerrow JH, Crack CS. The sequence, organization, and expression of the major cysteine protease (cruzain) from Trypanosoma cruzi. J Biol Chem 1992;267:7411-20.

24. Campetella O, Henriksson J, Aslund A, Frasch AC, Petterson U, Cazzulo JJ. The major cysteine proteinase (cruzipain) from Trypanosoma cruzi is encoded by multiple polymorphic tandemly organized genes located on different chromosomes. Mol Biochem Parasitol 1992;50:225-34.

25. Tomas AM, Kelly JM. Stage-regulated expression of cruzipain, the major cysteine protease of Trypanosoma cruzi is independent of the level of RNA1. Mol Biochem Parasitol 1996;76:91-103.

26. Lima AP, Tessier DC, Thomas DY, Scharfstein J, Storrer AC, Vernet T. Identification of new cysteine protease gene isoforms in Trypanosoma cruzi. Mol Biochem Parasitol 1994;67:333-8.

27. Lima AP, dos Reis FC, Serveau C, Lalmanach, Juliano L, Ménard R, et al. Cysteine protease isoforms from Trypanosoma cruzi, cruzipain 2 and cruzain, present different substrate preference and susceptibility to inhibitor. Mol Biochem Parasitol 2001;114:41-52.

28. Cazzulo JJ, Stoka V, Turk V. Cruzipain, the major cysteine proteinase from the protozoan parasite Trypanosoma cruzi. Biol Chem 1997;378:1-10.

29. Cazzulo JJ. Cruzipain, major cysteine proteinase of Trypanosoma cruzi: sequence and genomic organization of the codifying genes. Medicina (B Aires) 1999;59 (Suppl. 2):7-10.

30. Engel JC, Doyle PS, Hsieh I, McKerrow JH. Cysteine protease inhibitors cure an experimental Trypanosoma cruzi infection. J Exp Med 1998;188:725-34.

31. Klemba M, Goldberg DE. Biological roles of proteases in parasitic protozoa. Annu Rev Biochem 2002,71:275-305.

32. Del Nery E, Juliano MA, Lima AP, Schafstein J, Juliano $L$. Kininogenase activity by the major cysteinyl proteinase (cruzipain) from Trypanosoma cruzi. J Biol Chem 1997;272:25713-8.

33. Scharfstein J, Schmitz V, Morandi V, Capella MM, Lima AP, Morrot A, et al. Host cell invasion by Trypanosoma cruzi is potentiated by activation of bradykinin B(2) receptors. J Exp Med 2000;192:1289-300.

34. Aparicio IM, Scharfstein J, Lima AP. A new cruzipainmediated pathway of human cell invasion by Trypanosoma cruzi requires trypomastigote membranes. Infect Immun 2004;72:5892-902.

35. Berasain P, Carmona C, Frangione B, Cazzulo JJ, Goni F. Specific cleavage sites on human IgG subclasses by cruzipain, the major cysteine proteinase from Trypanosoma cruzi. Mol Biochem Parasitol 2003;130:23-9.

36. Camargo EP. Growth and differentiation in Trypanosoma cruzi. I. Origin of metacyclic trypanosomes in liquid media. Rev Inst Med Trop Sao Paulo 1964;12:93-100.

37. Miller SA, Dykes DD, Polesky HF. A simple salting out procedure for extracting DNA from human nucleated cells. Nucleic Acids Res 1988;16:1215.

38. Sambrook J, Fritsch EF, Maniatis T. Molecular cloning. A laboratory manual. Second edition. Cold Spring: Harbor Laboratory Press; 1989.

39. Xia X, Xie Z. DAMBE: software package for data analysis in molecular biology and evolution. $\mathrm{J}$ Hered 2001;92:371-3.

40. Rozas J, Sanchez-DelBarrio JC, Messeguer X, Rozas R. DnaSP, DNA polymorphism analyses by the coalescent and other methods. Bioinformatics 2003;19:2496-7

41. Carreño H, Rojas C, Aguilera X, Apt W, Miles MA, Solari A. Schizodeme analyses of Trypanosoma cruzi zymodemes from Chile. Exp Parasitol 1987;64:252-60.

42. Macedo AM, Pena SD. Genetic variability of Trypanosoma cruzi: implications for the pathogenesis of Chagas disease. Parasitol Today 1998;14:119-23.

43. Solari A, Wallace A, Ortiz S, Venegas J, Sánchez G. Biological characterization of Trypanosoma cruzi stocks from Chile. Exp Parasitol 1998;89:312-22.

44. Bogliolo AR, Lauria-Pires L, Gibson W. Polymorphisms in Trypanosoma cruzi: evidence of genetic recombination. Acta Trop 1996;61:31-40. 
45. Gaunt MW, Yeo M, Frame IA, Stothard JR, Carrascos HJ, Taylor MC, et al. Mechanism of genetic exchange in American trypanosomes. Nature 2003;421:936-9.

46. Brisse S, Henriksson J, Bernabé C, Douzery EJ, Berkvens D, Serrano M, et al. Evidence for genetic exchange and hybridization in Trypanosoma cruzi base on nucleotide sequences and molecular karyotype. Infect Genet Evol 2003;2:173-83.

47. Sturm NR, Vargas NS, Westenberger SJ, Zingales B, Campbell DA. Evidence for multiple hybrid groups in Trypanosoma cruzi. Int J Parasitol 2003,33:269-79.

48. Paulino M, Iribarne F, Dubin M, Aguilera-Morales S, Tapia O, Stoppani AO. The chemotherapy of Chagas' disease: an overview. Mini Rev Med Chem 2005;5:499-519.

49. Meiering S, Inhoff $\mathbf{O}$, Mies J, Vincek A, Garcia G, Kramer B, et al. Inhibitors of Trypanosoma cruzi trypanothione reductase revealed by virtual screening and parallel synthesis. J Med Chem 2005;48:4793-802.

50. Zimmer EA, Martin SL, Beverly SM, Kan YM, Wilson AC. Rapid duplication and loss of genes coding for the a chain of haemoglobin. Proc Natl Acad Sci USA 1980;77:2158-62.

51. Lynch M, Conery LM. The evolutionary fate and consequences of duplicate genes. Science 2000;290:1151-5

52. Hurles M. Gene duplication: the genomic trade in spare parts. PloS Biol 2004;2:E206.

53. Saravia NG, Holguín AF, Cibulskis RE, D'Alessandro A. Divergent isoenzyme profiles of sylvatic and domiciliary Trypanosoma cruzi in the Eastern plains, piedmont, and highlands of Colombia. Am J Trop Med Hyg 1987;36:59-69.
54. Cuervo P, Cupolillo E, Segura I, Saravia N, Fernandes $\mathbf{O}$. Genetic diversity of Colombian sylvatic Trypanosoma cruzi isolates revealed by the ribosomal DNA. Mem Inst Oswaldo Cruz 2002;97:877-80.

55. Jaramillo N, Moreno J, Triana O, Arcos-Burgos M, Muñoz S, Solari A. Genetic structure and phylogenetic relationships of Colombian Trypanosoma cruzi populations as determined by schizodeme and isoenzyme markers. Am J Trop Med Hyg 1999;61: 986-93.

56. Moncayo A. Chagas disease: current epidemiological trends after the interruption of vectorial and transfusional transmission in the Southern Cone countries. Mem Inst Oswaldo Cruz 2003;98:577-91.

57. Guhl F, Restrepo M, Angulo VM, Antunes CMF, Campbell-Lendrum D, Davies CR. Lessons from a national survey of Chagas disease transmission risk in Colombia. Trends Parasitol 2005;21:259-62.

58. Hartl CL, Clark AG. Principles of population genetics. Sunderland, Massachusetts; Sinauer Associate: 1997.

59. Garcia ES, Gonzalez MS, Azambuja P. Biological factors involving Trypanosoma cruzi life cycle in the invertebrate vector, Rhodnius prolixus. Mem Inst Oswaldo Cruz 1999;94(Suppl. 1):213-6.

60. Azambuja P, Garcia ES, Ratcliffe NA. Gut microbiota and parasite transmission by insect vectors. Trends Parasitol 2005;21:568-72.

61. Rodríguez P, Escalante M, Díez H, Cuervo C, Montilla M, Nicholls RS, et al. Estudio de la variabilidad de seis cepas colombianas de Trypanosoma cruzi mediante polimorfismos de longitud de los fragmentos de restricción (RFLP) y amplificación aleatoria de ADN polimórfico (RAPD). Biomédica 2002;22:263-71. 